have substantial latitude in defining minimum benefits for Medicaid and private insurance plans, including the breadth of prescription-drug coverage, which could substantially affect the quality of HIV care.

Although most HIV-infected patients should be better off, some will continue to fall through insurance-coverage cracks. Many immigrants are excluded from coverage provisions; paperwork and eligibility requirements may make it difficult for low-income patients to navigate the enrollment and reenrollment processes; and out-of-pocket costs for health insurance premiums and copayments may affect uptake and utilization of private insurance. Consequently, there will continue to be an important role for safety-net funding sources. However, the future of the Ryan White program and other discretionary safety-net programs that support the workforce of HIV care providers is uncertain; the current authorization for the Ryan White program expires in
2013. Major reductions in safetynet funding would make it difficult to support HIV-infected patients who cannot successfully navigate the new environment or afford subsidized insurance with sufficiently generous benefits to cover their care. Finally, the shift of funding from safetynet hospitals to community health centers may reduce capacity at HIV clinics affiliated with hospitals serving low-income patients.

The rationale for a grade A recommendation from the USPSTF is that there is "high certainty that the net benefit is substantial." In the case of HIV screening, that benefit can be achieved only if people identified as HIVinfected are effectively linked to and retained in HIV care and are supported in adhering to an effective antiretroviral regimen. The proposed USPSTF recommendations may remove financial barriers to routine HIV screening, but that is only the first step in ensuring that all HIV-infected Americans have access to the full continuum of care.
Disclosure forms provided by the authors are available with the full text of this article at NEJM.org.

From Rockefeller College of Public Affairs and Policy and the Nelson A. Rockefeller Institute of Government, University at AlbanyState University of New York, Albany (E.G.M.); and the Division of Health Policy, Department of Public Health, Weill Cornell Medical College, New York (B.R.S.).

This article was published on February 20, 2013, at NEJM.org.

1. Chou R, Selph S, Dana T, et al. Screening for HIV: systematic review to update the 2005 U.S. Preventive Services Task Force recommendation. Ann Intern Med 2012;157: 706-18.

2. Screening for HIV. Draft recommendation statement. Rockville, MD: Preventive Services Task Force, 2012 (http://www .uspreventiveservicestaskforce.org/uspstf13/ hiv/hivdraftrec.htm).

3. Branson B. Current HIV epidemiology and revised recommendations for HIV testing in health-care settings.J Med Virol 2007; 79:Suppl 1:S6-S10.

4. Martin EG, Schackman BR. What does US health reform mean for HIV clinical care? J Acquir Immune Defic Syndr 2012;60:72-6.

5. Martin EG, Paltiel AD, Walensky RP, Schackman BR. Expanded HIV screening in the United States: what will it cost government discretionary and entitlement programs? A budget impact analysis. Value Health 2010;13:893-902.

DOI: 10.1056/NEJMp1214630

Copyright @ 2013 Massachusetts Medical Society.

\title{
When to Start ART in Africa - An Urgent Research Priority
}

\author{
Kevin M. De Cock, M.D., D.T.M.\&H., and Wafaa M. El-Sadr, M.D., M.P.H.
}

\begin{abstract}
The history of the HIV-AIDS epidemic was profoundly altered by the introduction of antiretroviral therapy (ART). More than 8 million people in lowincome and middle-income countries have received lifesaving ART over the past decade, yet in 2011 an estimated 34 million people were living with HIV infection, 6.8 million were eligible for treatment but lacked access to ART, 2.5 million became newly infect-
\end{abstract}

ed, and 1.7 million died of HIVrelated disease. $^{1}$

Long-standing debate regarding the appropriate timing of ART initiation in the course of HIV infection was recently accentuated by the recognition of the prevention benefit that ART provides by reducing viral load and infectiousness. Mathematical models, ecologic analyses, and results from the HIV Prevention Trials Network (HPTN) study HPTN
052, a randomized, controlled trial that showed reduced HIV transmission from early, as compared with deferred, ART in the infected member of an HIV-discordant couple, ${ }^{2}$ all stimulated discussion of a "test and treat" approach, whereby all HIV-infected persons would initiate ART immediately after their HIV diagnosis, with anticipated reductions in transmission. A fundamental question remaining is what is best 


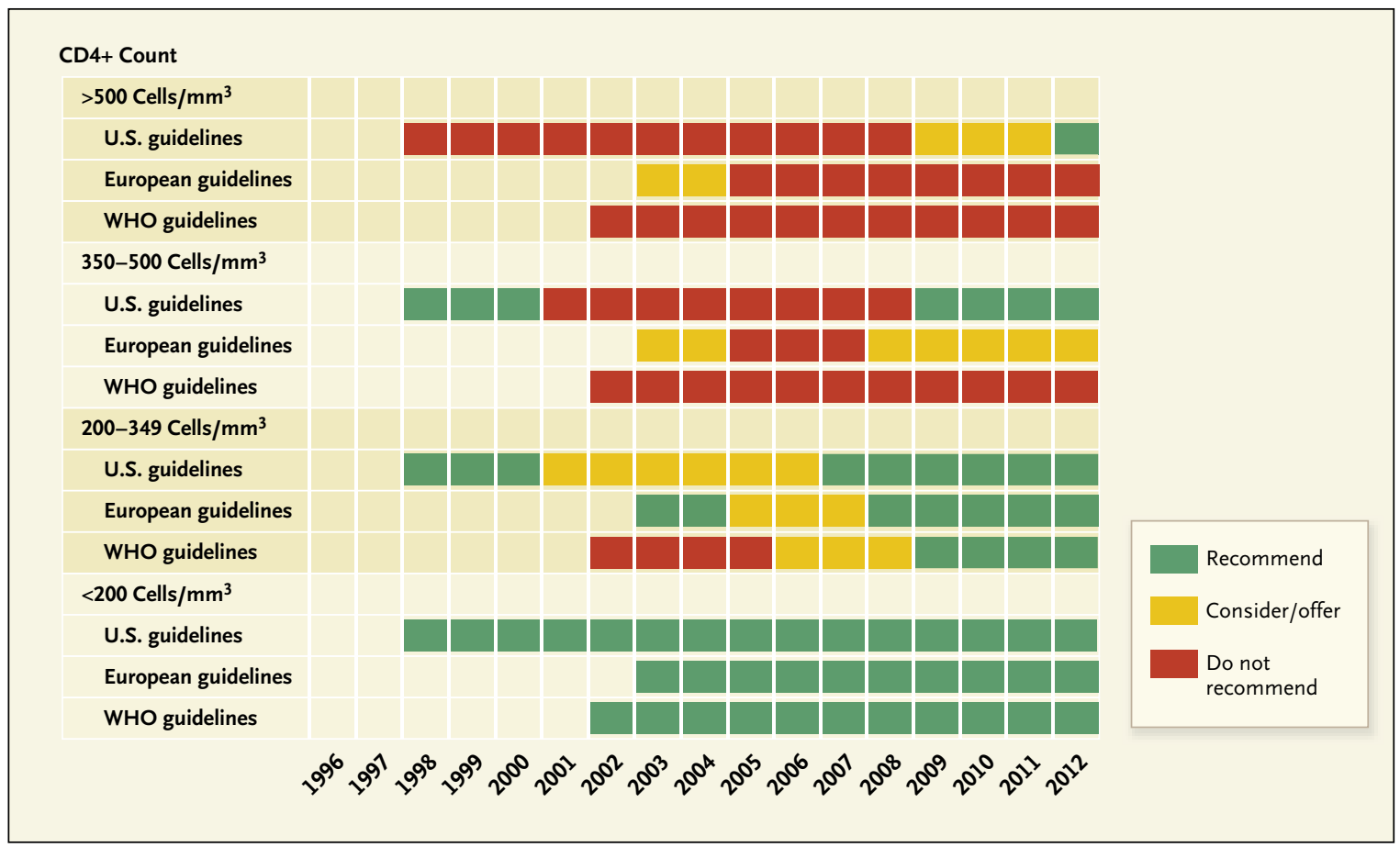

Evolution of CD4+ Count Criteria for Starting Antiretroviral Therapy in Asymptomatic Persons with Human Immunodeficiency Virus Infection, According to Different Guidelines.

Criteria for the United States are from the Department of Health and Human Services Guidelines for Use of Antiretroviral Therapy in Adults and Adolescents, those for Europe are from the European AIDS Clinical Society guidelines, and those for the World Health Organization (WHO) are from the WHO Antiretroviral Therapy Guidelines for Adults and Adolescents. Adapted from Marco Vitoria, M.D., of the World Health Organization.

for the health of the HIV-infected people who would take therapy for prevention, especially those in sub-Saharan Africa.

In the mid 1990s, we gained a clearer understanding of HIV replication and pathogenesis, viralload testing, and protease inhibitors, and studies showed the efficacy of triple ART in patients with HIV disease. "Hit early, hit hard" was a prevailing theme reflected in U.S. guidelines. Early therapeutic aggressiveness and optimism, however, became tempered by concerns about side effects and drug resistance, and the pendulum swung back toward guidelines more strongly supported by evidence from clinical trials and rigorous evaluations (see figure). For the first 6 years of this century, the Department of Health and Human Services (DHHS) recommended - on the basis of clinical-trial evidence - that ART be initiated when the CD4+ count dropped to less than 200 cells per cubic millimeter. As more durable and patient-friendly therapies were developed, this threshold was progressively raised and, largely on the basis of expert opinion, the 2012 DHHS guidelines essentially advocate treatment for anyone living with HIV infection. ${ }^{3}$

There is strong evidence and agreement that patients with CD4+ counts of less than 200 cells per cubic millimeter are at greatly increased risk for AIDS-related events and death and therefore urgently require ART. Since a ran- domized, controlled trial in Haiti showed reduced morbidity and mortality among persons in whom ART was initiated at a CD4+ count of 350 cells per cubic millimeter, as compared with those in whom therapy was deferred, ${ }^{4}$ that CD4+ level is now the minimum ART-initiation threshold globally, including under World Health Organization (WHO) guidelines. ${ }^{5}$

Some experts argue for even earlier initiation: full immunologic recovery lags if ART is unduly delayed, and uncontrolled HIV replication, irrespective of CD4+ count, results in immune activation and inflammation. These effects are probably causally related to many of the nonAIDS complications of HIV in- 
fection, such as cardiovascular disease and non-AIDS cancers, that are now the primary causes of death among HIV-infected people in high-income countries. Whether earlier ART would prevent such complications remains unknown. Results from observational studies examining the effect of early initiation on prevention of AIDS-related events and death are conflicting, and those investigations have focused solely on patients in industrialized countries. In addition to its primary aim of examining the prevention of HIV transmission in discordant couples, HPTN 052 compared clinical outcomes with early versus al Institutes of Health is supporting the Strategic Timing of Antiretroviral Treatment (START) study (ClinicalTrials.gov number, NCT00867048), in which HIVinfected persons with CD4+ counts of more than 500 cells per cubic millimeter are being randomly assigned to immediate ART or deferral until the count falls to 350 cells per cubic millimeter. The trial was designed to examine the effect of ART on noninfectious, non-AIDS complications of HIV infection that predominate in higher-income settings. Since relatively few patients from Africa are participating, it is unlikely that all uncertainties re-

\section{At least two thirds of people living with HIV infection are in sub-Saharan Africa. Debates about how best to use ART for both prevention and individual health are most relevant to the generalized HIV epidemics in this poorest geographic region.}

deferred therapy. However, it was not powered to examine mortality, the deferred-therapy group began receiving ART at $\mathrm{CD} 4+$ counts below the currently recommended threshold, and the clinical benefit that was observed was largely restricted to prevention of extrapulmonary tuberculosis. ${ }^{2}$

Uncertainty regarding the best approach to care for persons with higher CD4+ counts (>350 cells per cubic millimeter) is evident in the various guidelines that have been promulgated over the years (see figure). We need definitive data on which to base guidelines. To address the question of risks versus benefits of early ART initiation, the Nation- lated to African settings will be addressed.

At least two thirds of people living with HIV infection are in sub-Saharan Africa, where fragile health systems, health workforce shortages, weak laboratory infrastructure, and fiscal constraints are ubiquitous. Debates about how best to use ART for both prevention and individual health are most relevant to the generalized HIV epidemics in this poorest geographic region.

The spectrum of HIV disease varies internationally because of varying exposures and varying diagnostic and therapeutic capacity. Bacterial infections and tuberculosis dominate as causes of disease and death among HIVinfected people in Africa. The highest rate of adverse outcomes occurs among people with the lowest CD4+ counts, but the incidences of tuberculosis and bacterial infection are also increased at counts of 350 to 500 cells per cubic millimeter, and perhaps even at higher counts. Tuberculosis rates have increased by a factor of 5 to 10 in sub-Saharan Africa since the HIV-AIDS epidemic began. Mathematical modeling and a meta-analysis of treatment experience in resource-poor settings suggest that early ART could reduce the incidence of tuberculosis among patients with a wide range of $\mathrm{CD} 4$ counts, but conclusive data are lacking.

Early initiation of ART in Africa might also prevent HIV transmission. Mathematical models predict a large-scale effect from immediate ART use by all infected persons. Large-scale, community-based trials of combination preventive interventions, including the test-and-treat approach, are being planned, but unanswered questions about individual health benefits versus risks raise ethical and pragmatic issues. Should universal therapy be promoted for the common good of prevention, despite an uncertain health benefit for the person being treated? If immediate ART were shown to benefit individual health, the public health prioritization of the test-and-treat approach would be simplified, since it would be appropriate to offer diagnosis and immediate ART to everyone.

Demonstration of the dual benefit of ART has generated optimism about containing the HIV-AIDS pandemic. The diversity of clinical guidelines and practice, however, reflects a lack 
of definitive data indicating what is best for the persons who would be taking the drugs. The WHO characterizes the evidence available for informing the development of guidelines on ART initiation as weak, and changes in U.S. guidelines have been based largely on expert opinion.

We believe that a randomized, controlled trial should be undertaken immediately to determine when to initiate ART in Africa for maximal individual health benefit. It is feasible to conduct a large, simple trial to assess the risks and benefits of immediate ART versus deferral until a CD4+ count of 350 cells per cubic millimeter is reached. Key end points could be easy-to-ascertain events, such as tuberculosis incidence, hospitalization, and death. Equipoise would be present because of the conflicting evidence from observational studies, the absence of data from sub-Saharan Africa, limited data from randomized trials, divergent international practice, and inconsistent guidelines.
Uncertainty about ART is detrimental to the millions of people living with HIV infection. Early ART and deferred ART, each recommended and practiced depending on the setting, cannot both be the most favorable choice for individual health. To make informed decisions, HIV-infected people require a full understanding of the implications of taking ART early or late - or some may be reluctant to take early treatment for prevention. With millions of people who require ART still not receiving it, billions of dollars invested in HIV-AIDS programs, and widespread discussion about extending ART use, too much is at stake to allow uncertainty about when to initiate ART to persist.

The views expressed in this article are those of the authors and do not necessarily represent the official position of the Centers for Disease Control and Prevention or the Agency for Toxic Substances and Disease Registry.

Disclosure forms provided by the authors are available with the full text of this article at NEJM.org.
From the Centers for Disease Control and Prevention, Atlanta (K.M.D.C.); and ICAP, Columbia University Mailman School of Public Health, New York (W.M.E.-S.).

This article was published on February 20, 2013, at NEJM.org.

1. UNAIDS reports a more than $50 \%$ drop in new HIV infections across 25 countries as countries approach the 1000 day deadline to achieve global AIDS targets. Geneva: UNAIDS, 2012 (press release) (http://www .unaids.org/en/resources/presscentre/ pressreleaseandstatementarchive/2012/ november/20121120prresults/).

2. Cohen MS, Chen YQ, McCauley M, et al. Prevention of HIV-l infection with early antiretroviral therapy. N EnglJ Med 2011;365:493505.

3. AIDSinfo. Guidelines for the use of antiretroviral agents in HIV-l-infected adults and adolescents. Rockville, MD: Department of Health and Human Services, 2012 (http:// aidsinfo.nih.gov/contentfiles/lvguidelines/ AdultandAdolescentGL.pdf).

4. Severe P, Juste MAJ, Ambroise A, et al. Early versus standard antiretroviral therapy for HIV-infected adults in Haiti. N EnglJ Med 2010;363:257-65.

5. Antiretroviral therapy for HIV infection in adults and adolescents - recommendations for a public health approach: 2010 revision. Geneva: World Health Organization (http://www.who.int/hiv/pub/arv/adult2010/ en/index.html).

DOI: 10.1056/NEJMp1300458

Copyright @ 2013 Massachusetts Medical Society.

\title{
The Step 2 Clinical Skills Exam - A Poor Value Proposition
}

\author{
Elmer Philip Lehman IV, M.D., M.P.P., and Jason Ross Guercio, M.D., M.B.A.
}

Blatc fforts to minimize the regulatory and administrative burden in U.S. health care have never been greater. Stakeholders, including physicians, increasingly focus on eliminating waste and reducing costs in these areas to maximize the value and efficiency of care. One regulatory domain that deserves greater scrutiny in this context is medical licensure.

For example, the National Board of Medical Examiners (NBME) and the Federation of
State Medical Boards (FSMB) mandate the purchase of four licensing products by medical students and resident physicians over the course of their training. This three-step series of examinations is known as the United States Medical Licensing Examination (USMLE) and is jointly administered by the NBME and FSMB. The second exam, Step 2, is itself a two-part test administered in two sittings. One component, Step 2 Clinical Knowl- edge, is a multiple-choice exam; the other, Step 2 Clinical Skills (CS), is a day-long evaluation of clinical skills that takes place at one of five standardized-testing centers in the United States. Given the pass rates among examinees and the exam's cost, we believe that Step 2 CS provides a poor return on investment and little appreciable value to the U.S. health care system - and should therefore be eliminated.

The NBME and the FSMB 